\title{
Algunos aspectos fundamentales del número y la aritmética: una indagación cualitativa
}

\author{
Key aspects of the number and arithmetic: A qualitative inquiry \\ Mónica Angulo Cruz, Juan Pablo Marín Grisales, Gonzalo Díaz López \\ Departamento de Matemáticas, Universidad Tecnológica de Pereira, Pereira, Colombia \\ monaceutp.edu.co \\ jpmarin2004@yahoo.es \\ ghdl13@hotmail.com
}

\begin{abstract}
Resumen- El ser humano es un individuo producto de la naturaleza, y en su andar evolutivo ha tenido que sortear innumerables obstáculos a través de ayudas que le ha brindado su evolución. Muchas de estas ayudas evolutivas, han sido físicas $y$ motrices, pero también habilidades mentales, las cuales a través de ambientes de estimulación se han desarrollado para sostener al hombre a lo largo de la historia. Durante muchos años, diversos psicólogos e investigadores interesados en conocer el funcionamiento cognitivo del cerebro y de estas habilidades, han desarrollado experimentos que les han ayudado a ir revelando poco a poco esta actividad mental. Varios de estos experimentos se han enfocado en entender las capacidades innatas con las que los niños nacen que les permite interactuar con los demás seres humanos y con el entorno.
\end{abstract}

Palabras clave- habilidades, Pensamiento matemático, infancia, cerebro

Abstract - The individual human being is a product of nature, and in his walk evolution has had to overcome many obstacles through support extended to him their evolution. Many of these developmental aid, physical and motor have been, but also mental skills, which through stimulation environments have been developed to sustain man throughout history. For many years, various psychologists and researchers interested in learning about the cognitive functioning of the brain and these skills have developed experiments that have helped them go slowly revealing this mental activity. Several of these experiments have focused on understanding the innate abilities with which children are born that allows them to interact with other human beings and the environment.

Within these innate abilities, highlights the numeracy skills of elementary operations of addition and subtraction in children, which has been of great importance as well, can be a basis for an efficient and progressive development of thought mathematician in humans

Key Word - skills, mathematical thinking, childhood brain

Fecha de Recepción: 04 de Septiembre de 2013

Fecha de Aceptación: 30 de Abril de 2016

\section{INTRODUCCIÓN}

El presente documento invita a conocer paso a paso el trabajo investigativo que arrojó una visión diferente a los inicios de las habilidades aritméticas en los niños entre 0 y 2 años de edad. Busca confirmar y arrojar nueva información para entender que innatamente el ser humano nace, no sólo, con habilidades innatas, físicas, sino también con habilidades cognitivas que le permiten desarrollar todo su potencial a lo largo de su vida y que le dan el verdadero sentido a las matemáticas como una herramienta que hace parte además, de un progreso creado por el hombre, como también de un proceso de evolución que la naturaleza proporciona y viene en los genes.

\section{DESARROLLO DEL PENSAMIENTO NUMERICO}

Los niños en su infancia, cuentan con unas capacidades cerebrales ligadas a sus sentidos que no están completamente desarrolladas, pero si están listas para desplegarse rápidamente y consolidarse con los estímulos adecuados; Durante muchos años, numerosos científicos, centros investigativos y experiencias cotidianas, han generado una gran cantidad de información relativa a cómo evolucionan los bebés con el fin de convertirse en adultos por medio de procesos biológicos ${ }^{1}$ que son particulares de los seres humanos. La primera etapa cognoscitiva en la teoría de Piaget se denomina etapa Sensoriomotriz. Durante los dos primeros años de vida, los infantes aprenden de sí mismos y de su mundo a través de sus propias actividades sensoriales y motrices; los bebes se transforman, en criaturas que responden primordialmente por reflejos y comportamientos al azar, en infantes que dan sus primeros pasos orientados hacia una meta, organizan sus actividades en relación a su ambiente, coordinan la información que reciben de los sentidos y van del aprendizaje por ensayo y error al uso de

\footnotetext{
1 Procesos bilógicos son todas aquellas fases propias y naturales de los seres vivos, y por las cuales deben pasar para que se formen los cambios que implica su especie ( Salinas, N, 2005).
} 
estrategias rudimentarias para la solución de problemas simples. (Papalia, D y Wendkos S, 1997 p.135)

La Doctora Karen $\mathrm{Wynn}^{2}$ diseñó un experimento llamado "Evidencia en contra de las cuentas empiristas del origen del conocimiento numérico", el cual constituye la motivación inicial a la presente investigación, ya que por medio de este se logra vislumbrar que los bebes desde tempranas edades, tienen una capacidad innata para elaborar operaciones aritméticas elementales.

El experimento consiste en crear un pequeño escenario. En este se ubica una puerta delantera que se puede bajar y subir, cuando se sube, sirve para obstruir la visualización de cierta parte del escenario. Por una puerta lateral se pueden ingresar y retirar muñecos con la intención de formar conjuntos de estos, el ingresar implica hacer suma y el retirar implica hacer resta, así cuando se tiene un conjunto establecido de muñecos sobre el escenario y la puerta delantera se sube se ingresan o retiran los muñecos buscando que él bebe con el uso de su capacidad innata pueda predecir qué es lo que debe quedar en el escenario, al bajar la puerta él bebe confirma el resultado que predijo. Para conocer la certeza de la predictibilidad de los bebes, se miden sus reacciones ante situaciones ilógicas; las situaciones ilógicas se forman cuando él bebe observa que en una suma o resta el resultado no es el esperado, esto se logra retirando muñecos secretamente por una puerta trasera escondida en el escenario haciendo que los resultados sean ilógicos al momento de bajar la puerta. En el experimento desarrollado, estas situaciones se plantean para conjuntos de 2, 3, 4, 5 y hasta 6 muñecos con el fin de establecer hasta que cantidad de conjuntos los bebes tienen esta capacidad innata de suma y resta.

Según los resultados del experimento realizado por la doctora Wynn se encontraron que ante las situaciones de suma y resta que son de comportamiento lógico, los niños no se mostraban muy interesados, pero cuando se les mostraba situaciones ilógicas tanto de suma como de resta, estos tenían unas reacciones más notorias.

Dicho experimento va relacionado con un conjunto de teorías y definiciones que son valiosas en el presente documento ya que permite el análisis de los diferentes comportamientos que tienen los bebes, de igual forma contribuye a la comprensión de los datos obtenidos, logrando así la validación de algunas hipótesis planteadas desde el inicio de la investigación, tales como:

\footnotetext{
${ }^{2}$ Karen Wynn es una prestigiosa psicóloga que desde 1999, pertenece al Departamento de Psicología de la Universidad de Yale en donde ha realizado diversos experimentos sobre los procesos mentales que se dan en los niños y por los cuales interaccionan con el entorno, estos estudios le han valido diversos

reconocimientos a nivel mundial (Biografía Karen Winn.(sf))
}

- Los bebes mejoran progresivamente sus capacidades innatas de suma y resta a medida que sus sentidos se van estructurando.

- Los bebes cuando llegan a la edad de dos años poseen la capacidad innata de suma y resta con conjuntos de hasta 4 objetos.

\section{EXPERIMENTO DE KAREN WYNN}

Numerosos experimentos han mostrado que los bebes desde las primeras etapas de su vida tienen una relación innata con los números y las matemáticas. Los bebes desde los primeros días de su vida, pueden distinguir de una colección de 3 ó 4 objetos cuando estos se suman o restan entre sí. Si a un conjunto de 3 objetos se les quita 1 , los bebes pueden percibir este cambio sabiendo que deben de quedar 2 objetos, con lo cual están realizando un proceso aritmético de resta. Igualmente si a una colección de 1 objeto se le agrega otro, ellos alcanzan a percibir que deben quedar 2, con lo cual están sumando. Y estas mismas percepciones las pueden realizar con conjuntos de 3 ó 4 objetos.

Este fenómeno se ha descubierto por medio de un término psicológico llamado violación al nivel de expectativa, en el cual se observan los cambios en el comportamiento de un bebe cuando este se somete a situaciones diferentes a su cotidianidad o a la realidad.

Con esto, se ha llegado a demostrar, que los niños cuentan con una capacidad inicial y rudimentaria para realizar mentalmente operaciones aritméticas, las cuales se irán desarrollando a la par con su desarrollo mental (Lakoff y Núñez, 2000). El paso de llevar un sonido a un concepto es lento, pero estos son los pasos por los cuales el bebe comienza a moldear su cerebro para llegar a estos niveles de comprensión (Piaget, J, 1994).La Doctora Karen Wynn cuenta con una página web llamada "The Infant Cognition Center Yale University" en donde muestra los estudios que está realizando y varios aspectos de su amplio trabajo investigativo, todos relacionados con bebes.

En el trabajo de la doctora Wynn realiza experimentos de interacciones sociales en donde analizan si los bebes prefieren a las personas que son útiles a las personas que no muestran algún tipo de utilidad. Estudia la reciprocidad en donde trata de establecer que sensación tienen los bebes ante las personas que tratan bien a alguien y estas reciben igual trato. También estudia cómo los bebes comienzan a entender las expresiones faciales que muestran diferentes estados de ánimo. A pesar de que estos últimos trabajos no van relacionados con la matemática, la doctora Karen ha realizado estudios que son los motivadores de este trabajo.

\section{METODOLOGÍA}

Siendo el trabajo de campo el pilar fundamental para recoger la información y establecido por la práctica experimental con los niños dentro de los jardines se emplearon como técnicas de 
recopilación de datos: videos, entrevistas a las jardineras y fotografías. "Con todo lo que implica la recolección de los datos se establecerán las pautas para adelantar la investigación, en donde primará la profundidad y claridad de la información que se obtenga con claridad y certeza sobre las fuentes escrutadas" (Pulido, Ballén, Zúñiga, 2007, p. 53). La información recopilada por las diferentes técnicas fue introducida en plantillas diseñadas para clasificar y agrupar la información. Es así como se definen los parámetros de clasificación y codificación de la información para facilitar su debida observación y estudio de acuerdo a los objetivos planteados.

La compilación y organización de los datos extraídos en las entrevistas se realizaron de varias formas, se estableció qué materiales eran los más usados por los niños de acuerdo a su rango de edad y se estableció qué materiales eran los más representativos y los más usados por los niños. Por otro lado las actividades que los niños realizaban con estas herramientas también fueron muy importantes y se sistematizaron por cantidad de veces que se realizan y por rangos de edad, posteriormente se mostró cual era la actividad más frecuente de todas.

La apreciación de la jardinera para el aspecto matemático también se tomó en cuenta para saber qué actividades dentro de las que realizaban los niños consideraban ellas que definían en el niño un perfil matemático. Se compiló en forma general las actividades que realizaban los niños y cuáles eran las más representativas. Esta información igualmente se analizó mediante cuadros y gráficos. Se agrupó la información en rangos de edades de los 8 a los 12 meses, de los 12 a los 18 y de los 18 a los 24 meses.

Los cuadros mostraban distribuciones porcentuales en lo concerniente a los materiales con los que los bebes interactuaban, las prácticas que realizaban con estos, la apreciación de las jardineras de cada uno de los bebes y las actividades de los jardines relacionadas con las matemáticas.

La metodología empleada para analizar el dato hace referencia al análisis de contenido, el cual se basó en las fichas elaboradas de acuerdo a la información recogida por medio de grabaciones de videos o grabaciones de voz. Dicha información fue organizada y compilada para mostrar claramente lo que se observó y escuchó en las grabaciones y así poder analizar el contenido de estas.

La información de las fichas fue clasificada en forma resumida, compacta y sistemática, conformando en primera instancia unidades de contexto que "sirve de unidad para la comprensión de la unidad de registro" (Bardin, 1986, p.81), las cuales tienen la función de organizar la información en una frase que da significado y engloba todas aquellas expresiones de importancia investigativa y que son definidas como las unidades de registro "es un tipo de segmento textual claramente discernible" (Delgado, 1999 , p. 192), estas son palabras o frases clave que aclaran y dan relevancia a ciertos aspectos que dentro del análisis de los datos, arrojarán resultados que guían a las conclusiones.

Para las unidades de contexto se subdividieron las muestras en 3 rangos de edad que fueron: 8 a los 12 meses, de los 12 a los 18 meses y de los 18 a los 24 meses. Estas divisiones se usaron tanto para las fichas de los videos como para las fichas de las grabaciones de voz.

Para las unidades de registro se tomaron todas aquellas características observables y que mostraban notabilidad en el desarrollo del experimento con los niños, estas características se refieren a las diversas reacciones que se pudieron apreciar de estos. Igualmente en las grabaciones de voz o entrevistas propiamente dichas, se tomó como términos relevante aquellos elementos o acciones que se estimó tenían influencia sobre el tema investigado.

Toda esta organización de la información realizada para el análisis de contenido permitió conformar tablas donde los datos fueron congregados, y dada su relevancia podían ya ser más fácilmente identificables dentro del volumen de información que se tenía en las fichas anteriormente mencionadas.

"La fase del análisis propiamente dicha comienza por establecer unidades básicas de relevancia (de significación, en el sentido más amplio de la palabra) que el investigador se propone a extraer del corpus" (Delgado, 1999, p. 179).La información en bruto recopilada de acuerdo a cada formato establecido se encuentra disponible para ser estructurada y para su respectivo análisis. Teniendo en cuenta que:

La primera tarea de toda investigación empírica consiste en decidir qué se ha de observar y registrar, y lo que a partir de ese momento será considerado un dato. Hay buenas razones para utilizar el plural "datos" en lugar del singular, ya que toda investigación empírica abarca una multitud de unidades portadoras de información. La determinación de las unidades comprende su definición, su separación teniendo en cuenta sus respectivos límites y su identificación para el subsiguiente análisis (Krippendorff, 1990, p 81).

Se observan las categorías predeterminadas en este caso "suma" y "resta" y se procede a definir las categorías de análisis con las que se va a trabajar ya que "en el curso de un análisis concreto, el investigador puede crear sus propios esquemas de categorías, a partir de la información que le suministra su conjunto específico de datos" (Delgado, 1999, p. 195)

\section{ANALISIS DEL DATO}

El analizar y buscar respuestas implican que "Las situaciones problema proporcionan el contexto inmediato en donde el quehacer matemático cobra sentido, en la medida en que las 
situaciones que se aborden estén ligadas a experiencias cotidianas y, por ende, sean más significativas" (Ministerio de Educación Nacional, 2010). El problema que se quiere solucionar en esta investigación y precisamente en este análisis es identificar cuáles son las habilidades aritméticas innatas en niños entre 0 y 2 años de edad en la zona suroriental de la ciudad de Pereira, entendiendo como habilidades aritméticas innatas la capacidad de sumar y restar elementos de manera natural.

A continuación se realiza una descripción de los datos que se recogieron en las observaciones del trabajo de campo los que se encuentran registrados en fichas diseñadas para reunir la información, organizarla y facilitar su clasificación. Estas fichas compilan la información de manera sistemática por medio de unidades de contexto y unidades de registro de acuerdo al ejercicio de campo realizado y con base a criterios tales como rango de edad y cantidad de elementos utilizados para dichas pruebas.

Para llevar a cabo el análisis del dato se hace uso de la metodología análisis de contenido la cual "es una técnica destinada a formular, a partir de ciertos datos, inferencias reproducibles y válidas que puedan aplicarse a su contexto" (Krippendorff, 1990, p 28). El análisis de contenido busca proporcionar nuevos conocimientos, una representación de lo que está pasando y una guía para la acción (Krippendorff,k ,1990). En cuanto a la sume de operaciones lógicas de los 8 a los 12 meses de edad se presenta una distribución porcentual de las reacciones encontradas al evaluar la suma a través de operaciones lógicas mostrando una mayor proporción en las siguientes reacciones: "desvía su mirada" con un $60 \%$ y "observa atento y tranquilo" con un $20 \%$ frente a las demás reacciones encontradas.

Cada una de las reacciones encontradas en los niños en esta primera etapa, muestra que debido a su gran porcentaje en cuanto al tipo de manifestaciones que tienen los niños entre los 8 y los 12 meses de edad comprenden las operaciones que se están generando; al referirse a una operación lógica la reacción de desviar su mirada se ve como una acción tomada por el niño con la intención de mostrar desinterés ante lo que él ve, porque ya lo ha entendido y porque no le causa sorpresa. Este tipo de manifestaciones coincide con otros experimentos realizados con niños en donde esta reacción es la más típica cuando los niños se encuentran ante situaciones repetitivas y comunes.

Así mismo la reacción de "observa atento(a), tranquilo y concentrado(a)" es una reacción muy normal cuando no hay cambios en lo que se está observando y cuando se comprende lo que está pasando.

Las reacciones más notables por parte de los niños es cuando desvían su mirada al realizar el procedimiento con dos muñecos, lo que hace pensar y según teniendo presente investigaciones pasadas existe una mayor comprensión con la manipulación en un conjunto de dos muñecos. Sin embargo se evidencia que para un conjunto de tres muñecos la relación entre estas dos reacciones es muy uniforme lo que indica que los niños en este rango de edad pueden sumar de manera innata hasta un conjunto de tres muñecos con cierto detenimiento.

En cuanto a las reacciones encontradas en niños entre los 12 y 18 meses de edad en operación de suma lógica, se representa una distribución porcentual de las reacciones encontradas al evaluar la suma a través de operaciones lógicas, mostrando una proporción muy uniforme en las siguientes reacciones: "desvía su mirada" con un 39,29\% y "observa atento y tranquilo" con un $35,71 \%$ frente a otra muy cercana como lo es "cambia su expresión gestual (sonríe, serio) con $21,43 \%$.

Estas reacciones muestran debido a su gran porcentaje el tipo de manifestaciones que tiene un niño entre los 12 y los 18 meses de edad para expresar que entiende la operación que se está generando ya que al referirse a una operación lógicamente posible, la reacción de desviar su mirada se ve como una acción tomada por el niño con la intención de mostrar desinterés ante lo que él ve porque ya lo ha entendido y porque no le causa sorpresa. Este tipo de manifestaciones coincide con otros experimentos realizados con niños en donde esta reacción es la más típica cuando los niños se encuentran ante situaciones repetitivas y comunes.

Así mismo la reacción de "observa atento(a), tranquilo y concentrado(a)" es una reacción muy normal cuando no hay cambios en lo que se está viendo y cuando se entiende lo que está pasando. De igual manera los cambios de expresión son comunes cuando algo nos capta la atención. Pasar de la risa a la seriedad y viceversa, es un comportamiento que se presenta cuando no tenemos una explicación inmediata (sonrisa) y luego el tratar de buscarle una explicación, lleva al enfoque y concentración por el problema (seriedad)

Al revisar las reacciones para un conjunto de tres muñecos se observa que hay uniformidad en los resultados siendo estos muy similares lo cual indica que para este conjunto los niños ya entienden más la operación que se está generando. Sin embargo se evidencia que para un conjunto de cuatro muñecos hay valores muy aproximados en las siguientes reacciones: "desvía su mirada" y "observa atento y tranquilo" lo que da a pensar que los niños a esta edad poseen una aproximación con cierto grado de dificultad a la capacidad de suma de un conjunto de cuatro muñecos observando una transición y un desarrollo entre cada rango ya que al observar en la gráfica las reacciones cuando hay un conjunto de cinco muñecos estas se manifiestan en un bajo grado indicando que los niños no comprenden la operación para este conjunto.

Para la suma operaciones lógicas de los 18 a los 24 meses de edad se muestra una distribución porcentual de las reacciones encontradas al evaluar la suma a través de operaciones lógicas mostrando una proporción muy alta en la siguiente reacción: "observa atento y tranquilo" con un 47,62\%; Esta reacción 
muestran el tipo de manifestación que tiene un niño entre los 18 y los 24 meses de edad para expresar que entiende la operación.

Sin embargo se evidencia dos reacciones más las cuales tienen un porcentaje alto frente a las demás reacciones. La primera "cambia su expresión gestual (sonríe, serio)" con $14,29 \%$ y "mueve sus manos" con un $12,70 \%$ y cambia su expresión gestual con el mismo porcentaje, las cuales muestran otro tipo de acercamiento a la compresión de las operaciones lógicas de suma.

Al referirse a una operación lógica, la reacción de "observa atento(a), tranquilo y concentrado(a)" es una reacción muy normal notada en intervalos de tiempo cortos cuando no hay cambios en lo que se está viendo y cuando se entiende lo que está pasando.

De igual manera los cambios de expresión son comunes cuando algo nos capta la atención. Pasar de la risa a la seriedad y viceversa, es un comportamiento que se presenta cuando no tenemos una explicación inmediata (sonrisa) y luego el tratar de buscarle una explicación, lleva al enfoque y concentración por el problema (seriedad). El mover las manos es una reacción corporal que se genera ocasionalmente al estar pensando en una posible solución de un problema ya que genera inquietud y a la vez expectativa. De esta manera las anteriores reacciones indican que el niño comprende la operación lógica de suma en un determinado conjunto.

Las reacciones más notables "observa atento y tranquilo" y “cambia su expresión gestual (sonríe, serio)" y "mueve sus manos" muestran cuando el niño comprende el procedimiento en un conjunto de tres muñecos, indicando que en este rango de edad comprende las operaciones para este conjunto. Al revisar las reacciones para un conjunto de cuatro muñecos vemos que hay valores altos entre las reacciones de "cambia su expresión gestual" y "observa atento, tranquilo y concentrado" lo cual indica que el niño en este rango de edad comprende también operaciones de suma en un conjunto de cuatro muñecos ya que vemos que estas dos reacciones se complementan teniendo en cuenta que una, por su parte, muestra la atención del niño y la otra un comportamiento que se presenta cuando se lleva al enfoque y concentración por el problema (seriedad).

Se observó además que al analizar los resultados para un conjunto de cinco muñecos hay valores bajos pero uniformes en las reacciones más evidentes lo cual da a entender que los niños tendrían una cierta aproximación a la compresión de este tipo de operaciones en este conjunto. Sin embargo al avaluar la capacidad de suma para un conjunto de seis muñecos vemos que los valores son muy bajos y no se presentan mayores reacciones concluyendo que los niños a esta edad no entienden las operaciones para este tipo de conjuntos.
En cuanto la suma operaciones ilógicas de los 8 a los 12 meses de edad se muestra una distribución porcentual uniforme en las reacciones encontradas al evaluar la suma a través de operaciones ilógicas mostrando una mayor proporción en las siguientes reacciones: "desvía su mirada" con un 37,50\% y "centra fijamente su atención por más tiempo" con un 50\%, frente a "cambia su expresión gestual (sonríe, serio)" con $12,50 \%$. Teniendo en cuenta que se refieren a operaciones ilógicas la reacción de "centrar fijamente la mirada por más tiempo" coincide con otros experimentos realizados, donde esta reacción es la más típica cuando los niños se encuentran ante situaciones que se escapan a la percepción normal de su desarrollo ante diferentes fenómenos.

La reacción de desviar su mirada, se interpreta como una acción tomada por el niño con la intención de buscar un objeto que él considera perdido, ya que las operaciones de suma ilógicas efectuadas, generalmente mostraron un resultado donde faltaban elementos. Esta reacción es similar a cuando a un niño se le esconde un juguete, el niño percibe la falta de este y usando la capacidad de permanencia del objeto, rápidamente (no necesariamente en la primera vez) el niño comienza a tratar de resolver el hecho buscando el juguete en otros sitios diferentes.

Los cambios de expresión son comunes cuando algo llama la atención; pasar de la risa a la seriedad y viceversa, es un comportamiento que se presenta ante una situación en la que no se tiene una explicación inmediata (sonrisa) y luego el tratar de buscar una explicación, lleva al enfoque y concentración por el problema (seriedad).

Se puede concluir que las reacciones que representan mayor porcentaje muestran el tipo de reacciones manifestadas por los niños en este rango de edad ante situaciones ilógicas mostradas en la suma, y con lo cual se puede dilucidar que los niños entendían que algo estaba pasando.

Las reacciones más representativas son las reacciones más repetitivas en los niños cuando se les enfrenta ante operaciones ilógicas, en este caso para la suma. El desviar la mirada, muestra en el niño una reacción de búsqueda al encontrar que lo que sucedió en el escenario no fue lo que él esperaba y se desaparecieron muñecos que él esperaba ver. Esta reacción se puede ver en otros experimentos realizados los que buscan probar la capacidad de permanencia del objeto en donde, los niños al ver que un objeto que debería estar en cierta parte no se encuentra, comienzan a buscarlo en otros sectores, mostrando que estos mentalmente pueden mantener la imagen del objeto en donde debería estar.

Centrar la atención, es igualmente una reacción propia de los niños cuando observan situaciones que consideran ilógicas, estas son situaciones que dentro de sus sentidos no van de acuerdo a sus expectativas, por ello su reacción es observar el fenómeno con curiosidad buscando entender lo que sucede. La reacción de cambio en su expresión gestual, también muestra en el niño un cambio de actitud, este cambio de actitud se da normalmente 
cuando una situación no solo capta la atención y pasamos de la sorpresa a la búsqueda de explicación, sino que además la situación se torna extraña o curiosa, causando que se generen sensaciones de expectativa ante lo que se ve.

Las demás reacciones de "mira a su acompañante" con el $4,88 \%$, "mueve sus manos" con el 7,32\%, "parpadea" con el $4,88 \%$ e "inclina su cuerpo" con el $2,44 \%$, también se pueden interpretar como reacciones de sorpresa ante lo que acontece en el escenario, la poca frecuencia de estas reacciones puede deberse a que son reacciones ante situaciones ilógicas que van relacionadas con la propia aptitud del individuo, lo que quiere decir que son formas de reacción particulares en el niño que no necesariamente deben repetirse en otros. Por ejemplo inclinar el cuerpo hacia adelante es con la intención de buscar y tratar de ver hacia donde la situación ilógica se muestra y mirar al acompañante es una reacción de compartir la incertidumbre o de buscar explicación en otra persona.

Las reacciones de los niños ante las situaciones ilógicas de suma son notorias, destacándose notoriamente "centra fijamente su atención por más tiempo", "desvía su mirada" y "cambia su expresión gestual (sonríe, serio)". Los niños demuestran que estaban observando algo que no podían entender, evidenciando que en un conjunto de tres muñecos estas reacciones son más notorias lo cual da a pensar que el niño comprende cambios en este tipo de conjuntos. Sin embargo se ve que dentro de reacciones notorias como " centra su atención por más tiempo" y "desvía su mirada" hay valores amplios que indican que poseen una aproximación con cierto grado de dificultad a la capacidad de identificar que algo está pasando en una suma ilógica de un conjunto de cuatro muñecos, ya que al evaluar los resultados para un conjunto de cinco muñecos se observa que las reacciones evidentes se encuentran en un bajo porcentaje indicando que los niños no manifiestan actitudes de sorpresa ante diversos cambios.

\section{CONCLUSIONES}

El trabajo experimental realizado con los niños entre los 0 y los 2 años de edad en algunos jardines infantiles de la ciudad de Pereira permitió constatar que los niños cuentan con ciertas habilidades aritméticas innatas que hacen parte de su propia naturaleza y que además estas habilidades cuentan con un desarrollo progresivo en cada etapa de su vida.

A partir de la aplicación de las técnicas cualitativas y cuantitativas de la investigación y los diversos instrumentos de recolección de información como lo son las observación consignada en la fichas, las entrevistas, material audiovisual (videos y fotografías), datos obtenidos a partir de documentos, registros y el análisis de la información se concluye lo siguiente:

La suma es una operación que hace parte de las habilidades innatas de los niños en este rango de edad, ellos tienen la capacidad de diferenciar los cambios que se presentan en un conjunto de hasta tres muñecos y diferenciar que dos muñecos son más que uno y que tres muñecos son más que dos. Así mismo los niños tienen la capacidad de reaccionar ante operaciones ilógicas de suma en un conjunto de dos y tres muñecos, ya que en estas situaciones los niños muestran reacciones que indican que pueden percibir que dentro de la operación sucede algo que se sale de su lógica y que dicho resultado no es el que ellos esperaban.

Es importante resaltar que al evaluar la capacidad innata de suma en un conjunto de cuatro muñecos, las reacciones se evidencian en muy bajo grado lo cual indica que el niño en este rango de edad no comprende la operación de suma para este conjunto.

Siendo la resta la operación inversa de la suma, esta hace parte de las habilidades innatas encontradas en los niños en este rango de edad, pues los resultados muestran que niños entre los 8 y 12 meses de edad tienen la capacidad innata aritmética de restar hasta un conjunto de dos muñecos ya que se evidencian mayores reacciones y en un mayor grado en este tipo de conjuntos. Además al evaluar esta operación en un conjunto de tres muñecos las reacciones se muestran en un bajo grado lo cual indica que los niños no han desarrollado aun sus capacidades innatas aritméticas de resta para entender las operaciones realizadas en este tipo de conjuntos por lo cual el niño en este rango de edad solo puede distinguir que al quitar un muñeco de dos el resultado debe ser un solo muñeco o que al quitar dos muñecos de un conjunto de dos no queda ninguno.

Así mismo las operaciones ilógicas de resta realizadas con los niños para un conjunto de dos muñecos muestran que los niños reaccionan ante cambios inesperados en los resultados de un conjunto de dos muñecos. Ellos identifican el resultado como una situación anormal que se sale de su lógica y que por ejemplo al quitar un muñeco de dos, ellos esperarían ver un muñeco y no dos o tres como sucedió en algunos casos.

De esta manera es importante reiterar que los niños entre los 8 y los 12 meses de edad tienen la capacidad innata de sumar hasta un conjunto de tres muñecos y tienen la capacidad de restar hasta un conjunto de dos muñecos; así mismo que al enfrentar a los niños ante una operación de resta en un conjunto de tres muñecos los niños presentan cierta dificultad que no les permite interpretar correctamente esta operación, seguramente porque entre sus capacidades innatas solo cuentan con éstas.

Las habilidades innatas aritméticas en niños entre los 12 y los 18 meses de edad, se encuentra que ya tienen la capacidad innata de sumar hasta un conjunto de tres muñecos de manera más asertiva, pues sus habilidades innatas se muestran más 
desarrolladas. Al entrar a revisar las reacciones encontradas que determinan la capacidad innata aritmética de suma en conjuntos más grandes se evidencia que los niños entre los 12 y los 18 meses de edad pueden realizar operaciones innatas de suma hasta un conjunto de cuatro muñecos pues tanto en las operaciones lógicas como en las ilógicas para este conjunto los niños identifican los cambios en cantidad y los operan correctamente.

Al evaluar la capacidad de suma en un conjunto de cinco muñecos estas reacciones se manifiestan en un bajo grado indicando que los niños no comprenden la operación de suma para este conjunto y por lo tanto se afirma que los niños cuentan con la habilidad innata se suma hasta cuatro muñecos.

En este rango entre los 12 y los 18 meses de edad, los niños tienen una madurez cognitiva que les permite realizar innatamente operaciones de resta para un conjunto de tres muñecos evidenciando un avance el cual no se notaba en el anterior rango (entre los 8 y 12 meses). Hay un grado de dificultad que indica que en este rango de edad, solo en algunos casos, los niños pueden ejecutar correctamente una operación innata de resta para un conjunto de cuatro muñecos pues tanto en las operaciones lógicas como en las ilógicas las reacciones más notables no tienen un porcentaje muy alto.

Al evaluar la capacidad innata aritmética de resta en un conjunto de cinco muñecos se observa que las reacciones evidentes se encuentran en un bajo porcentaje, indicando que los niños no manifiestan actitudes de sorpresa ante diversos cambios y por lo tanto no entienden que pasa en este tipo de conjunto.

Se hace importante reiterar que los niños entre los 12 y los 18 meses de edad tienen la capacidad innata de sumar hasta un conjunto de tres y cuatro muñecos y que a la vez poseen la capacidad innata de restar hasta un conjunto de tres muñecos. Una vez reunidos los datos sobre las observaciones de las diversas reacciones en los niños de 18 a 24 meses de edad, se puede establecer un rango de conjunto sobre el cual se vislumbra que los niños tienen la habilidad innata de sumar.

Teniendo en cuenta los resultados obtenidos en el análisis de datos se identifica que los niños comprenden el procedimiento en un conjunto de tres muñecos, indicando que en este rango de edad comprende las operaciones para este conjunto. Los niños mostraron una capacidad para reconocer los cambios presentados en el ingreso de muñecos al escenario, concluyendo que podían sumar conjuntos de tres muñecos asimilando las diferentes formas con las que se mostrara la operación de suma.

Para un conjunto de cuatro muñecos vemos que hay valores altos entre las reacciones de "cambia su expresión gestual" y "observa atento, tranquilo y concentrado" lo cual indica que el niño en este rango de edad también comprende operaciones de suma en un conjunto de cuatro muñecos, ya que vemos que esta dos reacciones se complementan teniendo en cuenta que una por su parte muestra la atención del niño y la otra un comportamiento que se presenta cuando se lleva al enfoque y concentración por el problema (seriedad). Así mismo las operaciones ilógicas de suma se convierten en una prueba más para evidenciar que los niños perciben cambios en el ingreso de los muñecos al escenario pues, las reacciones son más notables y se visualizan de forma más clara. Cuando se les muestra a los niños operaciones de suma ilógicas con conjuntos de tres y cuatro muñecos ellos tienen reacciones muy notables, las cuales se interpretan como evidencia que afirman que para los niños pasaba algo que no iba de acuerdo a sus expectativas.

Es entonces donde para un conjunto de cinco, seis y siete muñecos los valores que representan las reacciones más evidentes son cada vez menores indicando que posiblemente tienen un acercamiento a entender los cambios para un conjunto de cinco muñecos pero, al aumentar la cantidad de muñecos, los niños no comprenden lo que está pasando y por lo tanto no manifiestan actitudes de sorpresa ante diversos cambios.

De acuerdo a lo anterior para los conjuntos de cinco, seis y hasta siete muñecos los resultados fueron un tanto disgregados y no tuvieron tanta consistencia como para los conjuntos de cantidad inferior, por lo cual se puede concluir que al menos para cinco muñecos algunos niños pueden percibir los cambios y tener una somera idea de un resultado esperado porque la habilidad innata está presente; por lo tanto innatamente los niños en este rango de edad tienen la capacidad aritméticas innata de sumar conjuntos de dos, tres, cuatro elementos y cinco elementos.

Con los datos recogidos en lo referente a la resta para los niños de los 18 a los 24 meses de edad, se pudo apreciar resultados coincidentes con el de la suma para los cuales los niños tienen la capacidad de restar en forma innata, pues se pudo apreciar que dichas reacciones señalaban que los niños podían visualizar las respuestas de los conjuntos de tres y cuatro muñecos coincidiendo con los resultados de suma mencionados anteriormente.

Se aprecia que para los conjuntos de tres y cuatro muñecos las reacciones más notorias se presentan con más facilidad por lo cual se puede establecer que procesan adecuadamente operaciones de resta lógica para estos conjuntos, así mismo se puede observar que para un conjunto de cinco muñecos los niños pueden alcanzar a interpretar este tipo de operación y distinguir la cantidad de elementos que deben quedar al hacer una resta con este conjunto.

Para un conjunto de cinco muñecos, se obtuvieron resultados con los cuales se pudo estimar que también tenían la capacidad innata de realizar operaciones con estos conjuntos, este hecho se articula con el de la suma, para el cual, algunos resultados mostraron capacidad de operar innatamente con esta cantidad de elementos y se une a la conclusión de que los niños, alcanzan a 
tener la capacidad innata de resta para cinco elementos. Por su parte, las operaciones ilógicas de resta confirman el potencial de evidencia en cuanto a la capacidad innata de resta que tienen los niños pues ellos pudieron mostrar varias reacciones notables que indicaban que algo fuera de lo normal estaba pasando, por lo cual, la resta con cuatro y cinco elementos se hace factible.

Las evidencias obtenidas para la resta ilógica, deja ver, que con cinco elementos un niño puede igualmente realizar operaciones de resta, las reacciones evidencian, que los niños perciben cambios al retirarse los muñecos y alcanzan a tener una visión mental de lo que debía quedar ya que en el conjunto de cinco elementos, adquieren un desarrollo en sus habilidades innatas de resta que les permite observar e identificar la operación que se está realizando para este conjunto, es decir, el niño puede identificar que se está realizando una operación de resta.

Dadas estas evidencias, se puede concluir que los niños en este rango de edad cuentan con una habilidad innata aritmética de suma y resta con tres, cuatro y hasta cinco elementos y que en general entre los 18 y 24 meses de edad, aun no tienen el desarrollo suficiente de sus habilidades innatas aritméticas para enfrentarse a operaciones de suma y resta en conjuntos superiores a cinco elementos pues al evaluar dichas reacciones en conjuntos más grandes no se evidencian suficientes reacciones que confirmen lo contrario.

\section{REFERENCIAS}

[1] Aritmética, Suma, Resta. García, R. (1978). Enciclopedia de la Ciencia. México: Editorial Larousse

[2] Arrau, A. (2003). Bases para la competencia en chile- la educación en una sociedad desigual. Santiago de Chile: RIL ®Editores.

[3] Baldin, L. (1986). Análisis de contenido. Madrid: Ediciones Akal S.A.

[4] Bardin L. (2002). Análisis de Contenido. Tercera Edición. Madrid España. Ediciones Akal.

[5] Bassedas, E.Huguet, T.(1998). Aprender y enseñar en educación infantil. Barcelona: Editorial GRAÓ, de IRIF, S.L.

[6] Beltran J., Bueno J. (1995). Psicología de la Educación. Barcelona España. Editorial Boixareu Universitaria.

[7] Conjuntos. Londoño,N. (1989).Serie Matemáticas Progresiva, Segunda edición. Colombia: Editorial Norma S.A
[8] Delgado, J. (1999). Métodos y técnicas cualitativas de investigación en ciencias sociales. Madrid: Editorial Síntesis S.A.

[9] Deslauriers, J. (2005). Investigación cualitativa guía práctica. Pereira Risaralda: Editorial Papiro.

[10]Díaz, R. (2009). Adquisición de la noción de número natural. Iberoamericana de Educación, 49/5, 9.

[11]Duque,L.(2007). Estadísticas sociales municipio de Pereira. Pereira. En prensa

[12] Gary, L. Augustowsky,G. Herr,k.Rivas,I. Suarez,D. Sverdlick,I.(2007). La investigación Educativa una herramienta de conocimiento y de acción. Argentina: Ediciones novedades educativas de México s.a. de C.V.

[13] Giráldez A. (2010). Música Investigación, innovación y buenas prácticas. Barcelona España. Editorial Graó.

[14] Gómez, J. (2004). Neurociencia Cognitiva y Educación, primera edición. Ciudad Universitaria de Lambayeque, Perú: Fondo Editorial FACHSE.

[15] González A., Ramos J. (2006). La Atención y sus Alteraciones: del Cerebro a la Conducta. Mexico. Editorial El Manual Moderno.

[16] Heinemann K. (2003). Introducción a la Metodología de la Investigación Empírica. Barcelona España. Editorial Paidotribo.

[17] Innato. Canda, F. (2002).Diccionario de Pedagogía y psicología. Madrid España: Cultural S.A

[18] Kostelnik M., Whiren A. (2009). Desarrollo social de los niños. Sexta Edición. Estados Unidos. Cengage Learning Editores.

[19] Krippendorff, K. (1990). Metodología de análisis de contenido. Barcelona: Ediciones Paidós Ibérica, S.A.

[20] M.luisa. Vasquez,N. Ferreira da Silva,R. Mogollón,A. de Sanmamed,J. Delgado,M. Vargas,I.(2006). Introducción a las Tecnicas Cualitativas de Investigación Aplicadas a la Salud. Barcelona: Servei de publicaciones-universidad autónoma de BarcelonaEspaña- Primera edición.

[21] Mayorga, C. (2002). Metodología de la investigación. Colombia: Editorial Panamericana.

[22] Mejía,R. Sandoval, S.(2003).Tras las vetas de la investigación cualitativa Tercera reimpresión. México: Instituto Tecnológico y de estudios Superiores de Occidente (Iteso).

[23] Ministerio de educación nacional. (1997) lineamientos curriculares Preescolar- Serie de documentos llamada "lineamientos curriculares". Bogotá.

[24] Morse, J. (2003). Asuntos críticos en los métodos de investigación cualitativa. Medellín: editorial universidad de Antioquia. 
[25] Salinas, N (2005). Manual para el técnico de sala de fitness. Barcelona: Editorial Paidotcibo.

[26] Núñez, L. (2000). Where Mathematics Comes From. Libro sobre un estudio de Investigación en Matemática Educativa sobre de donde vienen las matemáticas. New York: Basic Books

[27]Papalia, D. Wendkos, S. (1997). Desarrollo Humano Con Aportaciones para Iberoamérica sexta edición. Santafé de Bogotá: McGraw-Hill Interamericana, S.A.

[28]Patton.M.Q(1980). Qualitative evaluation Methods, Beverly Hills, California, Sage

[29] Piaget,J. (1965).La construcción de lo real en el niño, Tercera Edición. Buenos Aires Argentina: Editorial Proteo S.C.A

[30] Piaget,J. (1994).La formación del símbolo en el niño, Primera Reimpresión. Santa Fé de Bogota: Editorial Fondo de Cultura Económica, Ltda.

[31] Pulido,R. Ballen, M. Zuñiga, F.(2007). Abordaje hermeutico de la investigación cualitativa teorías, procesos técnicas. Bogotá: Editorial universidad cooperativa de Colombia.

[32]Rojas, R. (1987). Guía para realizar investigaciones sociales. Mexico: Editorial Plaza y Valdés S.A.

[33] Shaffer, D. (2000). Psicología del Desarrollo Infancia y Adolescencia. Quinta Edición. Buenos Aires Argentina. Editorial International Thomson Editores.

[34] Shelley, M.(2006). Terapia ocupacional en pediatría proceso de evaluación.Madrid: Editorial Médica Panamericana. S.A.

[35] Soler, P. (1997). La investigación cualitativa en el Marketing y publicidad. España: Ediciones Paidos, SAICF.

[36] Telmo,E.(2004).Revista latinoamericana de psicología año/vol.36 número 002.Bogota: Publicación Fundación universitaria Konrad Lorenz.

[37] Vasta, R. Haith, M y Miller, S. (1999). Psicología Infantil. Barcelona p. 318: Ariel.

[38]Zapata,O.(2005). La aventura del pensamiento crítico Herramientas para elaborar tesis e investigaciones Socioeducativas.Mexico D.C: Editorial Pax Mexico, librería Carlos Cesarman S.A. 